OPEN ACCESS

Edited by:

Marco Proietti,

University of Milan, Italy

Reviewed by:

Giulio Francesco Romiti,

Sapienza University of Rome, Italy

Pasquale Pignatelli,

Sapienza University of Rome, Italy

*Correspondence:

Chang-Sheng Ma

chshma@vip.sina.com

Specialty section:

This article was submitted to

Cardiac Rhythmology,

a section of the journa

Frontiers in Cardiovascular Medicine

Received: 21 August 2021

Accepted: 08 October 2021

Published: 18 November 2021

Citation:

Wang YF, Jiang C, He L, Du X, Sang

$C H$, Long DY, Tang RB, Dong JZ,

Lip GYH and Ma CS (2021) Integrated Care of Atrial Fibrillation Using the ABC (Atrial fibrillation Better Care)

Pathway Improves Clinical Outcomes in Chinese Population: An Analysis From the Chinese Atrial Fibrillation

\section{Integrated Care of Atrial Fibrillation Using the ABC (Atrial fibrillation Better Care) Pathway Improves Clinical Outcomes in Chinese Population: An Analysis From the Chinese Atrial Fibrillation Registry}

\author{
Yu-Feng Wang ${ }^{1}$, Chao Jiang ${ }^{1}$, Liu He ${ }^{1}$, Xin Du ${ }^{1,2,3}$, Cai-Hua Sang ${ }^{1}$, De-Yong Long ${ }^{1,4}$, \\ Ri-Bo Tang ${ }^{1}$, Jian-Zeng Dong ${ }^{1}$, Gregory Y. H. Lip ${ }^{5}$ and Chang-Sheng Ma ${ }^{\text {* }}$
}

${ }^{1}$ Department of Cardiology, Beijing AnZhen Hospital, Capital Medical University, National Clinical Research Centre for Cardiovascular Diseases, Beijing, China, ${ }^{2}$ Heart Health Research Centre, Beijing, China, ${ }^{3}$ The George Institute for Global Health, Faculty of Medicine, University of New South Wales, Sydney, NSW, Australia, ${ }^{4}$ Centre for Cardiovascular Diseases, The First Affiliated Hospital of Zhengzhou University, Zhengzhou University, Henan, China, ${ }^{5}$ Liverpool Centre for Cardiovascular Science, Liverpool Heart and Chest Hospital, University of Liverpool, Liverpool, United Kingdom

Background: "Atrial fibrillation Better Care" (ABC) pathway has been proposed to improve the management of patients suffered from atrial fibrillation (AF). This integrated or holistic management approach comprise of three aspects, including "A" Avoid stroke or Anticoagulation; "B" Better symptom control with rate or rhythm control strategies; "C" Cardiovascular risk factor and Concomitant diseases management. We aimed to confirm the beneficial evidence of ABC pathway compliance in a Chinese AF cohort.

Method and Results: From the Chinese Atrial Fibrillation registry (CAFR) dataset, a total of 19,187 non-valvular AF patients were enrolled, of which 4.365 (22.8\%) were ABC pathway compliant (ABC compliance group). During a median follow-up of $4.1 \pm$ 1.8 years, The incident rate of all-cause death in ABC compliance group and non-ABC compliance group is 2.7 and 1.1 per 100 person-year $(p<0.001)$, the incident rate of ischemic stroke is 1.3 and $0.8 \%$ per 100 person-year $(p<0.001)$, the incident rate of composite outcome, which consist of all-cause death, ischemic stroke and intracranial hemorrhage, is 3.8 and 1.9 per 100 person-year $(p<0.001)$. On Cox multivariable analysis, $\mathrm{ABC}$ pathway shows an independently association with reduction of all-cause death [hazard ratio $(\mathrm{HR})=0.82 ; 95 \%$ confidence interval $(\mathrm{Cl})=0.70-0.95$ ] and the composite outcome ( $\mathrm{HR} 0.86,95 \% \mathrm{Cl} 0.76-0.96)$. The increasingly components of $\mathrm{ABC}$ integrated care compliance is associated with lower risk of all-cause death and composite events. 
Conclusion: In a large cohort of Chinese AF patients, ABC pathway compliance shows an independently association with reduction of all-cause death and composite outcome of all-cause death, ischemic stroke and intracranial hemorrhage. Better compliance of $\mathrm{ABC}$ integrated care contributes to lower HR for adverse events.

Keywords: atrial fibrillation, $\mathrm{ABC}$ pathway, mortality, integrated care, comorbidity

\section{INTRODUCTION}

Atrial fibrillation (AF), which is a highly prevalent arrhythmia, has contributed to substantial cardiovascular death and cardiac morbidity from AF-related complications (1). Indeed, AF is associated with higher all-cause mortality ( $4.6 \%$ per year), only $10 \%$ of death is caused by stroke and nearly $50-60 \%$ attribute to other cardiovascular events $(2,3)$. Hence a comprehensive and integrated care method to management of AF and its associated comorbidities has been proposed to reduce these adverse outcomes $(4,5)$.

The Atrial fibrillation Better Care (ABC) pathway was introduced as an method to streamline the integrated or holistic management of AF (6). The ABC pathway has three main components: "A" refers to Avoid stroke with Anticoagulation, which means. optimizing stroke prevention with oral anticoagulation (OAC); "B" involves Better symptom management,i.e. improvement on symptom with rate or rhythm control by patient-centered symptom directed decisions; "C" applies to Cardiovascular risk factor and other Concomitant diseases management, including lifestyle modification (6).

Several studies have shown that the ABC pathway compliant management could reducing risk of adverse events (7-12) and this integrated pathway approach has also been recommended by 2020 ESC AF guidelines (13). Nonetheless, relatively few studies have investigated the impact of $\mathrm{ABC}$ pathway compliance in Asian countries, with the evidence derived from one cluster randomized trial (14) and one cohort study from Korea (9).

In China, nearly 7.9 million (about 2\%) people suffer from atrial fibrillation (15). In addition, the anticoagulated rate and comorbidities control rate are quite low (6 and $4 \%$ respectively) $(15,16)$, thus comprehensive management seems to be urgent for Chinese population. However, the beneficial evidence of this kind of pathway remains limited in Chinese population. In this analysis derived from the Chinese Atrial Fibrillation registry (CAFR) dataset, we investigated if $\mathrm{ABC}$ pathway compliance is associated with reduced adverse events in a large Chinese cohort consisting of AF patients.

\section{MATERIALS AND METHODS}

This retrospective analysis was based on the Chinese Atrial Fibrillation registry (CAFR), which is the largest, observational, prospective, register of AF patients in China. Detailed introduction about CAFR has been previously published (17). In short, the CAFR dataset has consecutively enrolled patients with AF from 31 tertiary and non-tertiary hospitals in Beijing, China, with regular follow-up of every 6 months for these patients. Pivotal data including demographic information, symptoms and signs related to $\mathrm{AF}$, other comorbidities and related medication or procedures, physical examination and biochemical test results will be collected at baseline. Severe adverse events comprising the occurrence of death and other cardiovascular diseases will also be collected at each 6 month by specialized follow-up team and adjudicated by professional endpoints committee.

Patients who were older than 18 years and diagnosed by AF episode of more than 30 second documented by 12-lead electrocardiogram were qualified for inclusion. Exclusion criteria were listed as follows: (1) valvular AF, including any mechanical bioprosthetic valves, or moderate to severe mitral stenosis, (2) those who had no baseline data up to 6 months before enrollment, and (3) those who had missing data such as European Heart Rhythm Association (EHRA) symptom score. After all these selection procedures, we enrolled non-valvular AF patients amount to 19,187 in this ancillary study to confirm the beneficial evidence of the $\mathrm{ABC}$ compliance on clinical events of AF patients.

The ethics committees of Beijing Anzhen Hospital has approved CAFR study and all patients involved in this study were informed about the detailed information and signed up for participating consent form.

\section{Definition of the ABC Pathway Compliant Group}

Based on the current clinical guidelines (13), the ABC pathway was defined as follows:

"A-Avoid stroke" - patients with a $\mathrm{CHA}_{2} \mathrm{DS}_{2}$-VASc score of 0 in men or 1 in women not receiving OAC and those with a $\mathrm{CHA}_{2} \mathrm{DS}_{2}$-VASc score of at least 2 in men or 3 in women anticoagulated were considered as compliant with the " $\mathrm{A}$ " criterion. We also considered patients with a $\mathrm{CHA}_{2} \mathrm{DS}_{2}$-VASc score of 1 in men or 2 in women as " $A$ " criterion compliant, no matter whether OAC were used. Others were recognized as "non-compliant" with the "A" criterion.

"B-Better symptom control"- Patients with no symptoms or mild symptoms having no influence on their daily life will be considered as EHRA I or II level. So "B compliance" refers to patients with EHRA score of I or II. Paitents with EHRA score of III or IV will be categorized as "non B compliance".

"C-Cardiovascular risk factor and other concomitant diseases optimization" - Cardiovascular diseases which is commonly associated with AF need to be treated with some specific medication or achieve standard goals. (1) for hypertension, baseline blood pressure $\leq 140 / 90 \mathrm{mmHg}$ was considered as well-controlled. (2) for heart failure, we considered treatment with ACE inhibitors/angiotensin receptor blockers 
and beta-blockers; (3) for coronary artery disease, treatment with angiotensin-converting enzyme (ACE) inhibitors, betablockers and statins; (4) for peripheral artery disease, treatment with statins; (5) for previous stroke/transient ischaemic attack, treatment with statins; (6) for diabetes mellitus, a fasting blood glucose of less than $7.0 \mathrm{mmol} / \mathrm{L}$ or glycosylated hemoglobin of less than $6.5 \%$ at baseline was considered as well-controlled. " $\mathrm{C}$ compliance" implies that all of these related concomitant diseases were either well-controlled or treated with suitable medication or both.

Paitents were identified as $\mathrm{ABC}$ pathway compliance if all of the components of $\mathrm{ABC}$ were fulfilled.

\section{Outcomes Definition}

The main events we assess in this study were all-cause motality, ischemic stroke, intracranial hemorrhage and a composite outcome of these three outcomes. Patients were followed from the index date until the study outcomes occurred or at end of follow-up, whichever occurred first. We compared these clinical events between $A F$ patients with $A B C$ pathway compliance (ABC compliance group) and those without this intergrated care (nonABC compliance group).

\section{Statistical Analysis}

Continuous variables were expressed as mean \pm standard deviation and compared by Student's $t$-test. Categorical variables were reported as frequency (percentage) and evaluated by Fisher's exact test or Pearson's chi-square test. We mainly analyzed the comparisons of clinical events between patients with and without $\mathrm{ABC}$ compliance. We also examined the association between total number of $\mathrm{ABC}$ compliance fulfilled and clinical events. We presented the cumulative incidences of clinical events by Kaplan-Meier curves and compared across the ABC and non$\mathrm{ABC}$ group with the log rank test. Cox multivariable regression

TABLE 1 | Baseline characteristics according to the ABC compliance.

\begin{tabular}{|c|c|c|c|c|}
\hline & $\begin{array}{l}\text { All patients } \\
N=19,187\end{array}$ & $\begin{array}{c}A B C \text { group } \\
N=43,65\end{array}$ & $\begin{array}{c}\text { Non-ABC group } \\
N=14,822\end{array}$ & $P$-value \\
\hline Age (years) & $64.2 \pm 12.0$ & $62.4 \pm 11.5$ & $64.7 \pm 12.1$ & $<0.0001$ \\
\hline Age $\geq 75, n(\%)$ & 4,187 (21.8) & $633(14.5)$ & $3,554(24.0)$ & $<0.0001$ \\
\hline Female, $n(\%)$ & $7,389(38.5)$ & $1,500(34.4)$ & $5,889(39.7)$ & $<0.0001$ \\
\hline $\mathrm{SBP}(\mathrm{mmHg})$ & $128.3 \pm 16.6$ & $120.0 \pm 10.2$ & $130.5 \pm 17.5$ & $<0.0001$ \\
\hline Body mass index $\left(\mathrm{kg} / \mathrm{m}^{2}\right)$ & $25.5 \pm 3.5$ & $25.5 \pm 3.6$ & $25.5 \pm 3.5$ & 0.0045 \\
\hline \multicolumn{5}{|l|}{ AF type, $n(\%)$} \\
\hline New onset & $1,179(6.1)$ & $229(5.3)$ & $950(6.4)$ & 0.0096 \\
\hline Paroxysmal & $10,982(57.2)$ & $2,490(57.0)$ & 8,492 (57.3) & \\
\hline Persistent/permanent & 7,026 (36.6) & $1,646(37.7)$ & 5,380 (36.3) & \\
\hline eGFR < 60 (CKD-EPI), $n$ (\%) & $1,876(9.8)$ & $224(5.1)$ & $1,652(11.2)$ & $<0.0001$ \\
\hline Current Smoking, $n$ (\%) & $2,253(11.7)$ & $602(13.8)$ & $1,651(11.1)$ & $<0.0001$ \\
\hline RFCA (baseline), $n$ (\%) & $8,644(45.1)$ & $1,910(43.8)$ & $6,734(45.4)$ & 0.0506 \\
\hline \multicolumn{5}{|l|}{ Comorbidities, $n$ (\%) } \\
\hline Coronary artery disease & 2,967 (15.5) & $121(2.8)$ & 2,846 (19.2) & $<0.0001$ \\
\hline Peripheral arterial disease & $153(0.8)$ & $10(0.2)$ & $143(1.0)$ & $<0.0001$ \\
\hline Hypertension & $12,250(63.9)$ & $2,231(51.1)$ & $10,019(67.6)$ & $<0.0001$ \\
\hline Heart failure & $2,719(14.2)$ & $187(4.3)$ & $2,532(17.1)$ & $<0.0001$ \\
\hline Ischemic stroke or TIA & $2,774(14.5)$ & $294(6.7)$ & $2,480(16.7)$ & $<0.0001$ \\
\hline Diabetes mellitus & 4,588 (23.9) & $570(13.1)$ & $4,018(27.1)$ & $<0.0001$ \\
\hline $\mathrm{CHA}_{2} \mathrm{DS}_{2}$-VASc, mean $\pm \mathrm{SD}$ & $2.5 \pm 1.8$ & $1.8 \pm 1.3$ & $2.7 \pm 1.9$ & $<0.0001$ \\
\hline HAS-BLED, mean \pm SD & $1.8 \pm 1.2$ & $1.5 \pm 0.9$ & $1.9 \pm 1.2$ & $<0.0001$ \\
\hline \multicolumn{5}{|l|}{ Medication, $n$ (\%) } \\
\hline Warfarin & $8,186(42.7)$ & 2,162 (49.5) & $6,024(40.6)$ & $<0.0001$ \\
\hline NOAC & $4,150(21.6)$ & $749(17.2)$ & 3,401 (23.0) & $<0.0001$ \\
\hline ACEI/ARB & $6,531(34.0)$ & 1,291 (29.6) & $5,240(35.4)$ & $<0.0001$ \\
\hline Beta-blocker & $8,144(42.5)$ & $1,790(41.0)$ & 6,354 (42.9) & 0.0288 \\
\hline Calcium channel blocker & $852(4.4)$ & $178(4.1)$ & $674(4.6)$ & 0.1858 \\
\hline Statin & $7,480(39.0)$ & $1,445(33.1)$ & $6,035(40.7)$ & $<0.0001$ \\
\hline Aspirin or Clopidogrel & 4,916 (25.6) & $802(18.4)$ & $4,114(27.8)$ & $<0.0001$ \\
\hline Insulin therapy or Oral antidiabetic drugs & $2,290(11.9)$ & $309(71)$ & $1,981(13.4)$ & $<0.00001$ \\
\hline
\end{tabular}

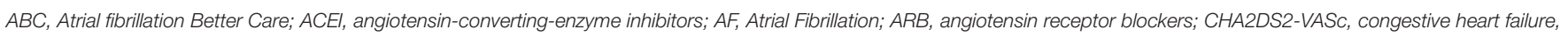

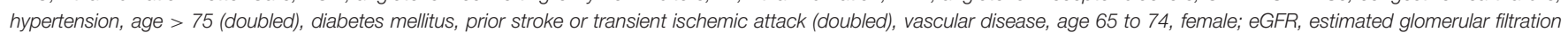

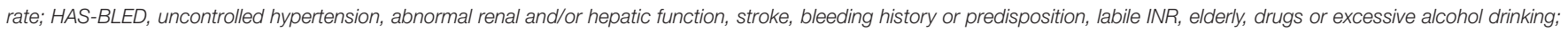
NOAC, non-vitamin K antagonist oral anticoagulant; SBP-systolic blood pressure; TIA, transient ischaemic attack. 
model was employed to analyze the hazard ratios (HRs) for clinical outcomes according to ABC compliance and adjusted clinical variables in this model includes age, sex, BMI, AF-type, eGFR $<60$, current smoking, RFCA, coronary artery disease, peripheral arterial disease, heart failure, hypertension, diabetes mellitus, and previous ischemic stroke/TIA. A two-side $p$-value of $<0.05$ was considered significant. All Statistical analyses were performed using SAS programming version 9.4.

\section{RESULTS}

Among 19,187 patients enrolled in this study from CAFR dataset, $22.8 \%(n=4,365)$ was defined as "ABC compliance group" and $77.2 \%(n=14,822)$ was identified as "Non-ABC compliance group". Baseline characteristic of these two groups were presented in Table $\mathbf{1 .}$

In comparison with non-ABC compliance group, the $\mathrm{ABC}$ compliance group were older $(62.4 \pm 11.5$ vs. $64.7 \pm 12.1, p<$ $0.001)$, less likely to be female $(34.3$ vs.38.7\% $p<0.001)$ and had a lower systolic blood pressure $(120.0 \pm 10.2$ vs. $130.5 \pm$ $17.5, p<0.001)$. The AF type in the ABC group was less likely to be new onset and paroxysmal AF. The ABC group also had a lower prevalence of comorbidities, including coronary artery disease, peripheral arterial disease, hypertension, heart failure, ischemic stroke or TIA, diabetes mellitus and chronic kidney disease (eGFR $<60$ ), and were treated with less drugs.

TABLE 2 | Compliance with the ABC pathway and its components.

\begin{tabular}{lcc}
\hline Study Groups & $\begin{array}{c}\text { ABC Compliant } \\
{[\mathbf{N}(\%)]}\end{array}$ & $\begin{array}{c}\text { Non-compliant } \\
{[\mathbf{N}(\%)]}\end{array}$ \\
\hline A & $12,499(65.1)$ & $6,688(34.9)$ \\
B & $14,021(73.1)$ & $5,166(26.9)$ \\
C & $9,347(48.7)$ & $9,840(51.3)$ \\
Hypertension & $6,034(49.4)$ & $6,189(50.6)$ \\
Heart failure & $562(33.8)$ & $1,101(66.2)$ \\
Ischemic stroke or TIA & $1,538(55.4)$ & $1,236(44.6)$ \\
Diabetes mellitus & $1,741(43.5)$ & $2,259(56.5)$ \\
Coronary artery disease & $740(25.0)$ & $2,219(75.0)$ \\
Peripheral arterial disease & $94(61.4)$ & $59(38.6)$ \\
All ABC & $4,365(22.8)$ & $14,822(77.2)$ \\
\hline
\end{tabular}

$A$, avoid stroke; $B$, better symptoms management; $C$, cardiovascular and other comorbidities; $A B C$, the full $A B C$ pathway compliance; TIA, transient ischaemic attack.
As expected, the $\mathrm{ABC}$ group had a lower mean $\mathrm{CHA}_{2} \mathrm{DS}_{2}$ VASc score $(1.8 \pm 1.3$ vs. $2.7 \pm 1.9, p<0.001)$ and HASBLED score $(1.5 \pm 0.9$ vs. $1.9 \pm 1.2, p<0.001)$ than the Non$\mathrm{ABC}$ group. There was a borderline significant difference in the proportion of catheter ablation use between these two groups $(p=0.0506)$.

\section{ABC Integrated Care Compliance}

Specific compliance of patients involved in this study toward each components of the ABC care is shown in Table 2, as follows: 12,499 (65.1\%) patients were managed in accordance with component "A", followed by rules of OAC use from the international guidelines; the " $\mathrm{B}$ " criterion was fulfilled in 14,021 (73.1\%) patients, who has no or only mild complain of clinical symptoms and was evaluated as EHRA I-II score due to the comprehensive management; "C" criterion-compliance, which refers to optimally treatment of associated concomitant diseases by specific medication or achievement of standard goals on the basis of international guidelines, was established in 9,347 (48.7\%). The compliance varied among these comorbidities, from peripheral arterial disease, up to $61.4 \%$, to coronary artery disease, only $25.0 \%$.

\section{Clinical Outcomes}

During a median follow-up of $4.1 \pm 1.8$ years. ABC pathway performed a lower rates of all-cause death (2.7 vs. 1.1 per 100 person-years, $p<0.001)$, ischemic stroke ( 1.3 vs. 0.8 per 100 person-years, $p<0.001$ ) and composite outcome (3.8 vs.1.9 per 100 person-years, $p<0.001)$ compared with no ABC compliance. Intracranial hemorrhage was not difference between these two groups ( 0.3 vs. 0.3 per 100 person-years, $p=0.8544$ ) (Table 3 ).

The cumulative incidence of clinical outcomes including all-cause death, ischemic stroke and composite outcome were significantly lower in $\mathrm{ABC}$ compliance group compared with the non-ABC compliance group. No difference was found in the cumulative incidence of intracranial hemorrhage (Figure 1).

Cox multivariable regression analysis showed $\mathrm{ABC}$ compliance was independently associated with a lower risk of all-cause death (hazard ratio [HR], 0.82; 95\% confidence interval [CI], 0.70-0.95) and composite outcome (HR, 0.86; 95\% CI, 0.76-0.96). No significant association with stroke and intracranial hemorrhage were found in patients with $\mathrm{ABC}$ compliance care (HR, 0.87; 95\% CI, 0.72-1.05 and HR, 1.22; 95\% CI, 0.87-1.72, respectively) (Table 4).

Subgroup analysis present a consistent result that $A B C$ compliance is associated with a lower risk of all cause death

TABLE 3 | Outcomes according to compliance with the ABC pathway.

\begin{tabular}{|c|c|c|c|c|}
\hline & $\begin{array}{l}\text { All patients } \\
N=19,187\end{array}$ & $\begin{array}{c}A B C \text { group } \\
N=4,365\end{array}$ & $\begin{array}{c}\text { Non-ABC group } \\
N=14,822\end{array}$ & $P$-value \\
\hline All-cause mortality, n (100 person-year) & $1,871(2.3)$ & $212(1.1)$ & $1,659(2.7)$ & $<0.0001$ \\
\hline Stroke, n (100 person-year) & $939(1.2)$ & $150(0.8)$ & 789 (1.3) & $<0.0001$ \\
\hline Intracranial hemorrhage, n (100 person-year) & $213(0.3)$ & $49(0.3)$ & $164(0.3)$ & 0.8544 \\
\hline Composite outcome, n (100 person-year) & $2,633(3.4)$ & $360(1.9)$ & $2,273(3.8)$ & $<0.0001$ \\
\hline
\end{tabular}




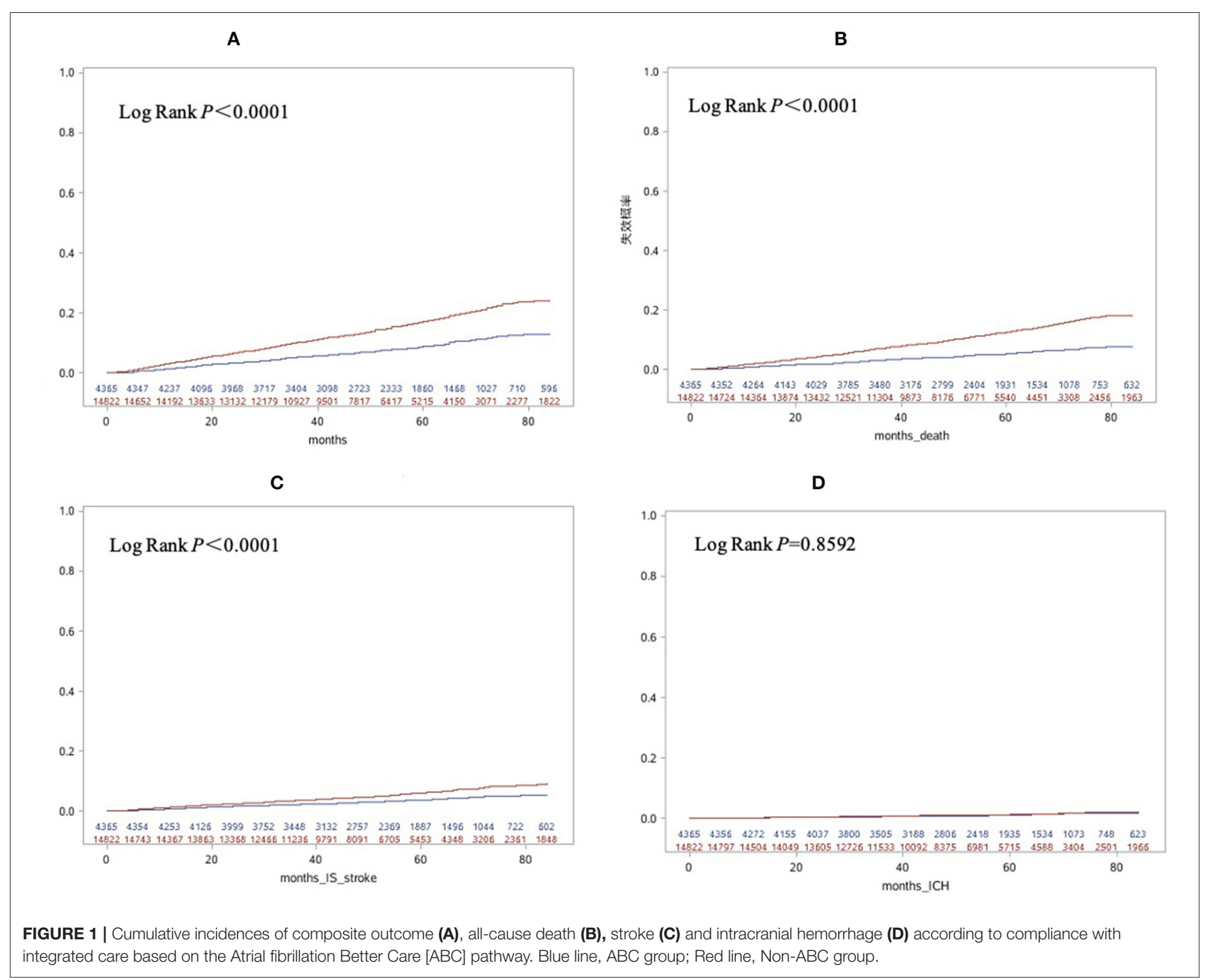

in patients with $\mathrm{AF}$ regardless of age, sex, $\mathrm{CHA}_{2} \mathrm{DS}_{2}$-VASC score, AF-type,eGFR $<60$, coronary artery disease, peripheral arterial disease, heart failure, hypertension, diabetes mellitus, and previous ischemic stroke/TIA (Figure 2).

\section{Number of ABC Criteria Fulfilled and Clinical Outcomes}

We examined the potential connection between fulfilled number of $\mathrm{ABC}$ component and adverse events. Adjusted Cox regression analysis showed that a higher numbers of $\mathrm{ABC}$ criteria fulfilled was independently associated with a progressively lower HRs of all-cause death and the composite outcome (Figure 3).

\section{DISCUSSION}

In this report from the CAFR Registry, we present novel data from a large observational cohort of Chinese AF patients, whereby clinical practice compliant with the $\mathrm{ABC}$ integrated care was associated with a significant lower risk in all-cause mortality
TABLE 4 | Regression models for the compliance of ABC in relation to outcomes.

\begin{tabular}{|c|c|c|c|c|}
\hline & \multicolumn{2}{|c|}{ Univariable } & \multicolumn{2}{|c|}{ Multivariable } \\
\hline & HR (95\% Cl) & $p$ Value & HR (95\% Cl) & $p$ Value \\
\hline $\begin{array}{l}\text { All-cause } \\
\text { mortality }\end{array}$ & $0.41(0.35,0.47)$ & $<0.0001$ & $0.82(0.70,0.95)$ & 0.0096 \\
\hline Stroke & $0.61(0.51,0.73)$ & $<0.0001$ & $0.87(0.72,1.05)$ & 0.1356 \\
\hline $\begin{array}{l}\text { Intracranial } \\
\text { hemorrhage }\end{array}$ & $0.97(0.70,1.33)$ & 0.8409 & $1.22(0.87,1.72)$ & 0.2468 \\
\hline $\begin{array}{l}\text { Composite } \\
\text { outcome }\end{array}$ & $0.50(0.45,0.56)$ & $<0.0001$ & $0.86(0.76,0.96)$ & 0.0102 \\
\hline
\end{tabular}

Hazard ratios are adjusted for age, sex, BMI, AF-type, eGFR < 60, Current Smoking, RFCA, Coronary artery disease, Peripheral arterial disease, heart failure, hypertension, diabetes mellitus, and previous ischemic stroke/TIA.

and composite outcome of all-cause death, ischemic stroke and intracranial hemorrhage. Moreover, with more components of 


\begin{tabular}{|c|c|c|c|}
\hline & & Hazard ratio $(95 \% \mathrm{CI})$ for all cause death & $\mathrm{P}$ for interaction \\
\hline \multicolumn{4}{|l|}{ Age } \\
\hline$<75$ & $0.92(0.76,1.13)$ & $\rightarrow-1$ & 0.61 \\
\hline$\geqslant 75$ & $0.74(0.58,0.94)$ & $\longmapsto \longrightarrow$ & \\
\hline \multicolumn{4}{|l|}{ Sex } \\
\hline Male & $0.75(0.62,0.91)$ & $\longmapsto$ & 0.14 \\
\hline Female & $0.96(0.75,1.23)$ & $\longrightarrow$ & \\
\hline \multicolumn{4}{|l|}{ AF type } \\
\hline Not persistent & $0.86(0.70,1.06)$ & 千 & 0.45 \\
\hline Persistent & $0.77(0.62,0.96)$ & $\longmapsto-1$ & \\
\hline \multicolumn{4}{|l|}{ eGFR $<60$} \\
\hline Yes & $0.66(0.43,1.00)$ & & 0.32 \\
\hline No & $0.84(0.71,0.99)$ & $\longmapsto$ & \\
\hline \multicolumn{4}{|c|}{ Coronary artery disease } \\
\hline Yes & $1.05(0.66,1.66)$ & $\longmapsto$ & 0.36 \\
\hline No & $0.82(0.70,0.97)$ & $\longmapsto$ & \\
\hline \multicolumn{4}{|l|}{ Hypertension } \\
\hline Yes & $0.81(0.67,0.98)$ & $\longmapsto$ & 0.88 \\
\hline No & $0.85(0.65,1.10)$ & $\longmapsto$ & \\
\hline \multicolumn{4}{|l|}{ Heart failure } \\
\hline Yes & $0.68(0.46,1.01)$ & - & 0.53 \\
\hline No & $0.89(0.75,1.05)$ & $\longmapsto$ & \\
\hline \multicolumn{4}{|c|}{ Ischemic stroke or TIA } \\
\hline Yes & $0.68(0.47,1.00)$ & $\rightarrow-$ & 0.58 \\
\hline No & $0.88(0.74,1.04)$ & $\longmapsto$ & \\
\hline \multicolumn{4}{|l|}{ Diabetes mellitus } \\
\hline Yes & $0.82(0.58,1.17)$ & $\longrightarrow$ & 0.36 \\
\hline No & $0.83(0.70,0.98)$ & $\longmapsto$ & \\
\hline \multicolumn{4}{|l|}{$\mathrm{CHA}_{2} \mathrm{DS}_{2}-\mathrm{VASC}$} \\
\hline $\mathrm{M}=0-1$ or $\mathrm{F}=1-2$ & $0.94(0.68,1,29)$ & $\longmapsto$ & 0.81 \\
\hline \multirow[t]{2}{*}{$\mathrm{M} \geq 2$ or $\mathrm{F} \geq 3$} & $0.83(0.69,0.99)$ & $\longmapsto$ & \\
\hline & $\begin{array}{ll} & \mathbf{r} \\
\text { ABC } & \mathbf{0 . 0} \\
\text { better }\end{array}$ & $T_{1.5}^{T}$ & $\begin{array}{r}\text { Non } \\
\text { be }\end{array}$ \\
\hline
\end{tabular}

FIGURE 2 | Forest plots presenting subgroup analysis for hazard ratio of all cause death for ABC compared with non-ABC group. Hazard ratios are adjusted for age, sex, BMI, AF-type, eGFR < 60, Current Smoking, RFCA, Coronary artery disease, Peripheral arterial disease, heart failure, hypertension, diabetes mellitus, and previous ischemic stroke/TIA.

ABC pathway criteria fulfilled, the risk of all-cause mortality and composite outcome was progressively lower.

The favorable impact resulting from comprehensive care and integrated method to AF management is reported in a randomized control trial and various real-world evidence studies (18, 19), although relatively limited data come from large Asian cohorts. The ABC pathway, which provides a simple, effective, and easily operationalizable integrated pr holistic AF management for patients and their carers, satisfies these demand and was 


\section{All-Cause Mortality}

The number of $A B C$ criteria fulfilled

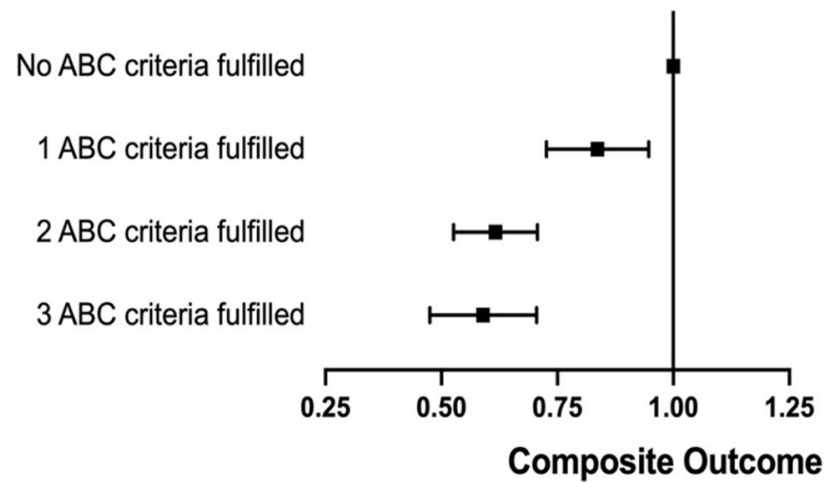

The number of $A B C$ criteria fulfilled

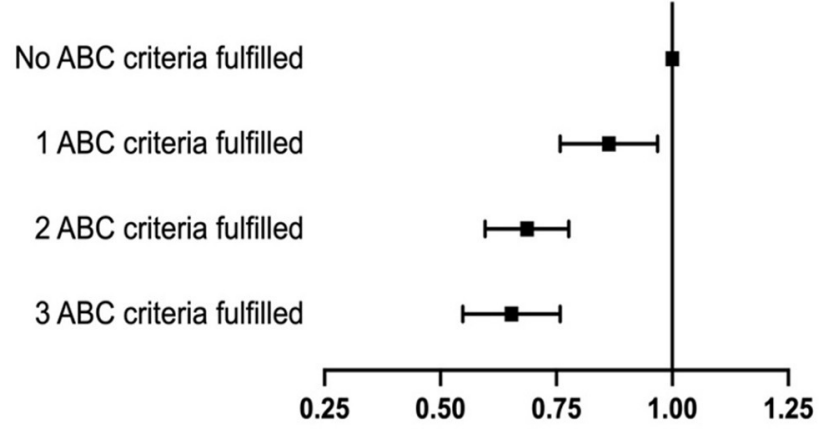

adjust $\mathrm{HR}(95 \% \mathrm{Cl}) \quad$ P Value

$\begin{array}{cc}\text { Ref. } & - \\ 0.83(0.73,0.95) & 0.0071 \\ 0.61(0.53,0.71) & <0.0001 \\ 0.58(0.48,0.71) & <0.0001\end{array}$

adjust $\mathrm{HR}(95 \% \mathrm{Cl}) \quad$ P Value

$\begin{array}{cc}\text { Ref. } & - \\ 0.86(0.77,0.97) & 0.0173 \\ 0.68(0.60,0.78) & <0.0001 \\ 0.65(0.55,0.76) & <0.0001\end{array}$

FIGURE 3 | Relationship between total number of ABC criteria fulfilled and outcomes.

recommended by the 2020 European Society of Cardiology guidelines (13).

Previous studies have shown that therapies compliant with the holistic care (ABC compliance group) were associated with a significantly lower all-cause death and the composite outcome consisting of cardiovascular motality, myocardial infarction, ischemic stroke, total hospitalizations and major bleeding, compared with non-ABC compliant group (7-12). Also, a system review and a meta-analysis have revealed an obvious risk reduction in $\mathrm{ABC}$ group despite the variation including population selection, definition of $\mathrm{ABC}$ and clinical outcomes among these studies $(20,21)$. Our findings are consistent with the foregoing studies presenting lower hazard of all-cause death and the composite outcome in patients compliant with $\mathrm{ABC}$ care in comparsion to those without $\mathrm{ABC}$ pathway. The result that the risk of all-cause mortality and composite outcome was progressively lower with more components of $\mathrm{ABC}$ pathway criteria fulfilled was also found in other similar studies, which further points to an important lesson that a larger clinical benefit would accrue to those who were increasingly adherent to the ABC pathway management $(7,9,10)$.

In our study, Cox regression did not present a significant lower risk in ischemic stroke and intracranial hemorrhage, and the similar result was also reported in other studies, including a recent randomized controlled trial $(7,10,14)$. We hypothesized that these results may be caused by the relatively low incidence of stroke and intracranial hemorrhage events compared with other cohort studies, and the poor control of anticoagulation observed from Asian cohorts (22, 23) as nearly $50 \%$ of AF patients were prescribed warfarin in our study.

As the original cohort, from which the data were derived, represents the largest observational study for AF management in China, the results we presented are indicative of contemporary AF management nationwide in comparsion to previous studies exploring the beneficial evidence of ABC compliance approach that confined in limited cohorts, or those which were involved with patients from other countries (7-9). Moreover, this cohort comprises of the largest number of patients with $\mathrm{ABC}$ compliance management, presenting reliable results in relation to the amount of patients and events.

\section{LIMITATIONS}

As an observational study, there are some limitations to be considered. Almost half of AF patients using warfarin 
in this cohort, and the lack of TTR data remains the efficiency of anticoagulation unknown, leading to some bias of the correlation of $\mathrm{ABC}$ adherent care and clinical outcomes. Even though the multivariable COX regression analysis presented an significant association between $A B C$ compliance and reduced risk of these adverse events, some other related factors were not involved, including the presence of other concomitant diseases not available at baseline in this study or mentioned as part of the "C" criterion. Also, we have limited information on individual adherence and persistence to medication as well as the lack of a formalized approach to the evaluation of pharmacological treatment.

Finally, given the nature of the observational study, our data could only describe associations rather than demonstrate causality. Even if a large number of covariates have been adjusted in the the multivariable analysis, it seems to be unreasonable to make an inference of causality with the differences at baseline characteristics between the $\mathrm{ABC}$ group and non- $\mathrm{ABC}$ group. Hence, prospective studies with adequate power are needed to further confirm our findings.

\section{CONCLUSIONS}

In a large cohort of Chinese $\mathrm{AF}$ patients, $\mathrm{ABC}$ integrated management presents a significant association with a reduced risk for all-cause motality and composite outcome of all-cause death, ischemic stroke and intracranial hemorrhage. With higher number of the components of $\mathrm{ABC}$ compliance fulfilled, the risk of clinical events was progressively lower.

An increasing number of $\mathrm{ABC}$ criteria fulfilled was associated with a progressively lower risk of adverse clinical outcomes.

\section{REFERENCES}

1. Odutayo A, Wong CX, Hsiao AJ, Hopewell S, Altman DG, Emdin CA. Atrial fibrillation and risks of cardiovascular disease, renal disease, and death: Systematic review and meta-analysis. BMJ. (2016) 354:i4482. doi: 10.1136/bmj.i4482

2. Gomez-Outes A, Lagunar-Ruiz J, Terleira-Fernandez AI, Calvo-Rojas G, Suarez-Gea ML, Vargas-Castrillon E. Causes of death in anticoagulated patients with atrial fibrillation. J Am Coll Cardiol. (2016) 68:250821. doi: 10.1016/j.jacc.2016.09.944

3. Fauchier L, Villejoubert O, Clementy N, Bernard A, Pierre B, Angoulvant $\mathrm{D}$, et al. Causes of death and influencing factors in patients with atrial fibrillation. Am J Med. (2016) 129:1278-87. doi: 10.1016/j.amjmed.2016. 06.045

4. Kirchhof P. The future of atrial fibrillation management: integrated care and stratified therapy. Lancet. (2017) 390:187387. doi: 10.1016/S0140-6736(17)31072-3

5. Kotecha D, Breithardt G, Camm AJ, Lip GYH, Schotten U, Ahlsson A, et al. Integrating new approaches to atrial fibrillation management: the 6th afnet/ehra consensus conference. Europace. (2018) 20:395-407. doi: 10.1093/europace/eux318

6. Lip GYH. The abc pathway: an integrated approach to improve af management. Nat Rev Cardiol. (2017) 14:6278. doi: $10.1038 /$ nrcardio. 2017.153

\section{DATA AVAILABILITY STATEMENT}

The original contributions presented in the study are included in the article, further inquiries can be directed to the corresponding author.

\section{ETHICS STATEMENT}

The studies involving human participants were reviewed and approved by the Ethics Committees of Beijing Anzhen Hospital. The patients/participants provided their written informed consent to participate in this study.

\section{AUTHOR CONTRIBUTIONS}

Y-FW wrote this paper with help of other professors, including making study design, analyzing data, and revising this article. All authors contributed to the article and approved the submitted version.

\section{FUNDING}

This work was supported by the National Key Research and Development Program of China (Grant Numbers: 2018YFC1312501 and 2017YFC0908803) and the National Natural Science Foundation of China (82103904). The construction of the Chinese Atrial Fibrillation Registry was also supported by grants from Bristol-Myers Squibb, Pfizer, Johnson \& Johnson, Boehringer-Ingelheim, and Bayer.

\section{ACKNOWLEDGMENTS}

This study was based on data from CAFR. We would like to thank the CAFR investigators for assistance in the data collection.

7. Proietti M, Lip GYH, Laroche C, Fauchier L, Marin F, Nabauer M, et al. Relation of outcomes to abc (atrial fibrillation better care) pathway adherent care in european patients with atrial fibrillation: An analysis from the esc-ehra eorp atrial fibrillation general long-term (afgen lt) registry. Europace. (2021) 23:174-83. doi: 10.1093/europace/euaa274

8. Gumprecht J, Domek M, Proietti M, Li YG, Asaad N, Rashed W, et al. Compliance of atrial fibrillation treatment with the atrial fibrillation better care $(\mathrm{abc})$ pathway improves the clinical outcomes in the middle east population: a report from the gulf survey of atrial fibrillation events (safe) registry. J Clin Med. (2020) 9:1286. doi: 10.3390/jcm9051286

9. Yoon M, Yang PS, Jang E, Yu HT, Kim TH, Uhm JS, et al. Improved population-based clinical outcomes of patients with atrial fibrillation by compliance with the simple abc (atrial fibrillation better care) pathway for integrated care management: a nationwide cohort study. Thromb Haemost. (2019) 119:1695-703. doi: 10.1055/s-0039-1693516

10. Proietti M, Romiti GF, Olshansky B, Lane DA, Lip GYH. Improved outcomes by integrated care of anticoagulated patients with atrial fibrillation using the simple abc (atrial fibrillation better care) pathway. Am J Med. (2018) 131:1359-66 e1356 doi: 10.1016/j.amjmed.2018.06.012

11. Pastori D, Pignatelli P, Menichelli D, Violi F, Lip GYH. Integrated care management of patients with atrial fibrillation and risk of cardiovascular events: the abc (atrial fibrillation better care) pathway in the athero-af study cohort. Mayo Clin Proc. (2019) 94:1261-7. doi: 10.1016/j.mayocp.2018. 10.022 
12. Proietti M, Romiti GF, Olshansky B, Lane DA, Lip GYH. Comprehensive management with the abc (atrial fibrillation better care) pathway in clinically complex patients with atrial fibrillation: a post hoc ancillary analysis from the affirm trial. J Am Heart Assoc. (2020) 9:e014932. doi: 10.1161/JAHA.119.014932

13. Hindricks G, Potpara T, Dagres N, Arbelo E, Bax JJ, Blomstrom-Lundqvist C, et al. 2020 esc guidelines for the diagnosis and management of atrial fibrillation developed in collaboration with the european association for cardio-thoracic surgery (eacts). Eur Heart J. (2021) 42:373-498. doi: 10.1093/eurheartj/ehaa612

14. Guo Y, Lane DA, Wang L, Zhang H, Wang H, Zhang W, et al. Mobile health technology to improve care for patients with atrial fibrillation. J Am Coll Cardiol. (2020) 75:1523-34. doi: 10.1016/j.jacc.2020.01.052

15. Du X, Guo L, Xia S, Du J, Anderson C, Arima H, et al. Atrial fibrillation prevalence, awareness and management in a nationwide survey of adults in china. Heart. (2021) 107:535-41. doi: 10.1136/heartjnl-2020-317915

16. Jiang C, Lan DH, Du X, Geng YP, Chang SS, Zheng D, et al. Prevalence of modifiable risk factors and relation to stroke and death in patients with atrial fibrillation: a report from the china atrial fibrillation registry study. J Cardiovasc Electrophysiol. (2019) 30:2759-66. doi: 10.1111/jce. 14231

17. Du X, Ma C, Wu J, Li S, Ning M, Tang R, et al. Rationale and design of the chinese atrial fibrillation registry study. BMC Cardiovasc Disord. (2016) 16:130. doi: 10.1186/s12872-016-0308-1

18. Hendriks JM, de Wit R, Crijns HJ, Vrijhoef HJ, Prins MH, Pisters R, et al. Nurse-led care vs. usual care for patients with atrial fibrillation: results of a randomized trial of integrated chronic care vs. routine clinical care in ambulatory patients with atrial fibrillation. Eur Heart J. (2012) 33:26929. doi: 10.1093/eurheartj/ehs071

19. Nieuwlaat R, Olsson SB, Lip GY, Camm AJ, Breithardt G, Capucci A, et al. Guideline-adherent antithrombotic treatment is associated with improved outcomes compared with undertreatment in high-risk patients with atrial fibrillation. The euro heart survey on atrial fibrillation. Am Heart J. (2007) 153:1006-12. doi: 10.1016/j.ahj.2007.03.008

20. Stevens D, Harrison SL, Kolamunnage-Dona R, Lip GYH, Lane DA. The atrial fibrillation better care pathway for managing atrial fibrillation: a review. Europace. (2021) 23:1511-27. doi: 10.1093/europace/ euab092
21. Romiti GF, Pastori D, Rivera-Caravaca JM, Ding WY, Gue YX, Menichelli $\mathrm{D}$, et al. Adherence to the 'atrial fibrillation better care' pathway in patients with atrial fibrillation: impact on clinical outcomes-a systematic review and meta-analysis of 285,000 patients. Thromb Haemost. (2021). doi: 10.1055/a-1515-9630. [Epub ahead of print].

22. Singer DE, Hellkamp AS, Piccini JP, Mahaffey KW, Lokhnygina Y, Pan G, et al. Impact of global geographic region on time in therapeutic range on warfarin anticoagulant therapy: data from the rocket af clinical trial. J Am Heart Assoc. (2013) 2:e000067. doi: 10.1161/JAHA.112.000067

23. Shimada YJ, Yamashita T, Koretsune Y, Kimura T, Abe K, Sasaki S, et al. Effects of regional differences in asia on efficacy and safety of edoxaban compared with warfarin-insights from the engage af-timi 48 trial. Circ J. (2015) 79:2560-7. doi: 10.1253/circj.CJ-15-0574

Conflict of Interest: C-SM has received honoraria from Bristol-Myers Squibb, Pfizer, Johnson \& Johnson, Boehringer-Ingelheim, and Bayer for giving lectures. J-ZD has received honoraria from Johnson \& Johnson for giving lectures. GL has served as a Consultant and Speaker for BMS/Pfizer, Boehringer Ingelheim, and Daiichi-Sankyo.

The remaining authors declare that the research was conducted in the absence of any commercial or financial relationships that could be construed as a potential conflict of interest.

Publisher's Note: All claims expressed in this article are solely those of the authors and do not necessarily represent those of their affiliated organizations, or those of the publisher, the editors and the reviewers. Any product that may be evaluated in this article, or claim that may be made by its manufacturer, is not guaranteed or endorsed by the publisher.

Copyright (c) 2021 Wang, Jiang, He, Du, Sang, Long, Tang, Dong, Lip and Ma. This is an open-access article distributed under the terms of the Creative Commons Attribution License (CC BY). The use, distribution or reproduction in other forums is permitted, provided the original author(s) and the copyright owner(s) are credited and that the original publication in this journal is cited, in accordance with accepted academic practice. No use, distribution or reproduction is permitted which does not comply with these terms. 